\section{P146 COGNITIVE LOSS IN STABLE NON-HYPOXAEMIC PATIENTS WITH MODERATE CHRONIC OBSTRUCTIVE PULMONARY DISEASE (COPD)}

doi:10.1136/thx.2010.150987.47

${ }^{1} \mathrm{~J}$ W Dodd, ${ }^{2} \mathrm{M}$ Van Den Broek, ${ }^{3} \mathrm{D}$ Shrikrishna, ${ }^{3} \mathrm{~N}$ S Hopkinson, ${ }^{1} \mathrm{P}$ W Jones. ${ }^{1} \mathrm{St}$ George's University of London, London, UK; ${ }^{2}$ St George's Hospital NHS Trust, London, UK; ${ }^{3}$ Royal Brompton \& Harefield NHS Trust, London, UK

Background Cognitive impairment has been demonstrated in up to $77 \%$ of patients with COPD and hypoxaemia, ${ }^{1}$ however it is unclear if this impairment is due to cognitive loss or was present before disease onset. In addition the evidence for impairment is less clear in patients with mild disease without hypoxaemia.

Methods Detailed neuropsychological testing of 44 non-exacerbating, non-hypoxaemic patients with COPD. Other physiological, and health status data were collected. Standardised neuropsychological scores were generated from age, gender and education related normative population samples. Cognition is considered moderately or severely impaired if a standardised score lie $\geq 1$ or 2 standard deviations (SD) respectively from the normative population mean. The use of population normal range is valid given that the group have a normal estimated Full Scale IO. Estimates of an individuals predicted cognitive performance in attention and processing speed are derived from their adult reading ability, which is stable over time and largely resistant to cognitive decline. Individuals performing below their predicted performance are judged to have suffered cognitive loss. This methodology has advantages over control group comparisons since the individual acts their own control allowing for calculations of what is 'normal' for that individual.

Results 44 patients, $52 \%$ Females, Mean Age $68 \pm 8$ years, $F_{1 \mathrm{pp}}$ $49 \pm 20 \%, \mathrm{PaO}_{2} 10 \pm 2 \mathrm{kPa}$, Pack Years. smoking 57 (41-69), 6MWD 387m (359-458), BMI 26 (23-29), SGRO 52 \pm 19 , Hospital Anxiety and Depression Scale 12 (6-19), Fatigue (FACIT-F) 18 (9-25), Estimated Full Scale IO 96. In terms of cognitive loss, $25 \%$ had significant loss in processing speed and 30\% in working memory. The proportion of patients whose cognitive scores were 1 and 2 SD's below predicted are tabulated.

Conclusion Up to one third of stable non-hypoxaemic patients with COPD demonstrate significant cognitive loss. A significant proportion have severe impairment of memory and executive function. The cause and implications of this cognitive loss should be the focus of future research.

\begin{tabular}{lll}
\hline $\begin{array}{l}\text { Cognitive } \\
\text { function }\end{array}$ & $\begin{array}{l}\text { Moderate impairment } \\
\text { ( } \geq \text { 1SD below mean) }\end{array}$ & $\begin{array}{l}\text { Severe impairment } \\
\text { ( } 2 \text { 2SD below mean) }\end{array}$ \\
\hline Memory & $66 \%$ & $32 \%$ \\
Attention & $48 \%$ & $5 \%$ \\
Processing speed & $50 \%$ & $5 \%$ \\
Executive & $73 \%$ & $50 \%$ \\
Visuospatial & $32 \%$ & $16 \%$ \\
\hline
\end{tabular}

\section{DEPRIVATION IS ASSOCIATED WITH INCREASED HEALTHCARE UTILISATION IN PATIENTS WITH CHRONIC OBSTRUCTIVE PULMONARY DISEASE}

doi:10.1136/thx.2010.150987.48

${ }^{1} \mathrm{P} F$ Collins, ${ }^{1} \mathrm{R}$ J Stratton, ${ }^{2} \mathrm{R}$ Kurukulaaratchy, ${ }^{1} \mathrm{M}$ Elia. ${ }^{1}$ Institute of Human Nutrition, School of Medicine, University of Southampton, Southampton, England; ${ }^{2}$ Respiratory Medicine, Southampton University Hospital NHS Trust, Southampton, England

Deprivation assessed using the index of multiple deprivation (IMD) has been shown to be an independent risk factor for 1-year mortality in outpatients with chronic obstructive pulmonary disease; COPD (Collins et al, 2010). IMD combines a number of economic and social issues (eg, health, education, employment) into one overall deprivation score, the higher the score the higher an individual's deprivation. Whilst malnutrition in COPD has been linked to increased healthcare use it is not clear if deprivation is also independently associated. This study aimed to investigate the influence of deprivation on 1-year healthcare utilisation in outpatients with COPD. IMD was established in 424 outpatients with COPD according to the geographical location for each patient's address (postcode) and related to their healthcare use in the year post-date screened (Nobel et al, 2008). Patients were routinely screened in outpatient clinics for malnutrition using the 'Malnutrition Universal Screening Tool', 'MUST' (Elia 2003); mean age 73 (SD 9.9) years; body mass index 25.8 (SD 6.3) kg/m ${ }^{2}$ with healthcare use collected 1 year from screening (Abstract P147 Table 1). Deprivation assessed using IMD (mean 15.9; SD 11.1) was found to be a significant predictor for the frequency and duration of emergency hospital admissions as well as the duration of elective hospital admission. Deprivation was also linked to reduced secondary care outpatient appointment attendance but not an increase in failure to attend and deprivation was not associated with increased disease severity, as classified by the GOLD criteria $(p=0.580)$. COPD outpatients residing in more deprived areas experience increased hospitalisation rates but decreased outpatient appointment attendance. The underlying reason behind this disparity in healthcare use requires further investigation. Collins P.F. et al, (2010) presented at BTS winter meeting (SP165). Stratton RJ, et al, (2003) Disease-related malnutrition, CABI publishing; Elia M (Ed.) (2003) The 'MUST' report. BAPEN, Redditch (www.bapen. org.uk); Nobel et al, (2008) The English indices of deprivation 2007 (www.communities.gov.uk)

Abstract 147 Table1

\begin{tabular}{|c|c|c|c|}
\hline 1-year healthcare use $(n=424)$ & Mean (SD) & $\beta$-coefficient & \\
\hline & & * & $p$ \\
\hline No. of emergency hospital admissions & $0.62(1.5)$ & 0.24 & $<0.001$ \\
\hline Emergency length of hospital stay (days) & $4.9(13.2)$ & 1.5 & 0.015 \\
\hline No. of elective hospital admissions & $0.27(0.75)$ & 0 & 0.842 \\
\hline Elective admission length of hospital stay (days) & $1.1(7.2)$ & 1.2 & 0.001 \\
\hline No. of secondary care outpatient appointments & $3.7(2.9)$ & -4.1 & 0.002 \\
\hline
\end{tabular}

\section{REFERENCE}

1. Grant, et al. Arch Intern Med 1982;142:1470.
Funding Funded by an unrestricted educational grant from Nutricia. 